\title{
Radio Channel Characterization in Dense Forest Environments for IoT-5G ${ }^{+}$
}

\author{
Peio Lopez-Iturri 1,2, Erik Aguirre 1,2, Mikel Celaya-Echarri ${ }^{3}$, Leyre Azpilicueta ${ }^{3}$, \\ Alejandro Eguizábal ${ }^{1}$, Francisco Falcone ${ }^{1,2}$ and Ana Alejos ${ }^{4, *}$ \\ 1 Department of Electric, Electronic and Communication Engineering, Public University of Navarre, 31006 \\ Pamplona, Navarra, Spain; peio.lopez@unavarra.es (P.L.-I.); erik.aguirre@unavarra.es (E.A.); \\ alex.egui.2@gmail.com (A.E.); francisco.falcone@unavarra.es (F.F.) \\ 2 Institute for Smart Cities, Public University of Navarre, 31006 Pamplona, Navarra, Spain \\ 3 School of Engineering and Sciences, Tecnologico de Monterrey, 64849 Monterrey, Mexico; \\ mikelcelaya@gmail.com (M.C.-E.); leyre.azpilicueta@itesm.mx (L.A.) \\ 4 Department of Signal theory and Communications, University of Vigo, 36310 Vigo, Spain \\ * Correspondence: analejos@uvigo.es; Tel.: +34-986-811-949 \\ + Presented at the 5th International Electronic Conference on Sensors and Applications, 15-30 November \\ 2018; Available online: https://ecsa-5.sciforum.net.
}

Published: 14 November 2018

\begin{abstract}
The attenuation due to vegetation can limit drastically the performance of Wireless Sensor Networks (WSN) and the Internet of Things (IoT) communication systems. Even more for the envisaged high data rates expected for the upcoming $5 \mathrm{G}$ mobile wireless communications. In this context, radio planning tasks become necessary in order to assess the validity of future WSN and IoT systems operating in vegetation environments. For that purpose, path loss models for scenarios with vegetation play a key role since they provide RF power estimations that allow an optimized design and performance of the wireless network. Although different propagation models for vegetation obstacles can be found in the literature, a model combining path loss and multipath propagation is rarely considered. In this contribution, we present the characterization of the radio channel for IoT and 5G systems working at $2.4 \mathrm{GHz}$, focusing on the radio links blocked by oak and pine trees modelled from specimens found in a real recreation area located within a dense forest environment. This specific forest, composed of thick in-leaf trees, is called Orgi Forest and it is situated in Navarre, Spain. In order to fit and validate a radio channel model for this type of scenarios, both measurements and simulations by means of an in-house developed 3D Ray Launching algorithm have been performed, offers as outcomes the path loss and multipath information of the scenario under study. A geometrical and dielectric model of the trees were created and introduced in the simulation software. The path loss was then estimated as dependent of the radio link range for two species of trees at $2.4 \mathrm{GHz}$. We concluded that the scattering produced by the tree can be divided into two zones with different dominant propagation mechanisms: a freespace zone far from the tree and a diffraction zone around the edge of the tree. 2D planes of delay spread value are also presented which similarly reflects the proposed two-zone model.
\end{abstract}

Keywords: Internet of Things; wireless sensor networks; vegetation; 5G; radio channel model

\section{Introduction}

Inhomogeneous vegetation environments have the special feature of acting as scatterers of electromagnetic waves. The signal scattering is translated into an excess of attenuation which can limit the performance of the Internet of Things (IoT) envisaged at high data rates and low latency expected for the upcoming $5 \mathrm{G}$ mobile wireless communications. In this context, radio planning tasks become necessary in order to assess the validity of future IoT systems operating in vegetation 
environments. For that purpose, path loss models for scenarios with vegetation play a key role since they provide RF power estimations that allow an optimized design and performance of the wireless network [1]. Moreover, a path loss model may contribute to evaluate the maximum effective distance between adjacent terminals, hence to estimate the number of sensors needed to cover a certain area. Finally, the signal strength loss is related to the quality of service (QoS), causing unreliable communication between nodes that will increase both the number of data packet retransmissions and the power consumption of the nodes, causing radio link failure in last term. Therefore, there is a need for reliable through-vegetation radio channel modelling for vegetation environments, which will assess the propagation behaviour in terms of both path loss and multipath propagation.

The characterization of the radio channel blocked by vegetation elements has been largely studied in literature, which proposed different models to estimate the power attenuation or level excess loss introduced by a signal blockage due to vegetation obstacles, mainly trees [2-4]. However, a model combining path loss and multipath is rarely considered. In this contribution, we present a simple model to characterize the attenuation due to the isolated thin trees in an air-to-air communication channel occurring between a static transmitter and a mobile user which moves linearly toward the tree. The developed channel model also considers parameters due to the multipath presence obtaining the value of the delay spread parameter.

The radio characterization was performed by means of simulations based on 3D Ray Launching software, where the specific material parameters of the vegetation elements are considered, such as dielectric constant and conductivity. The paper is organized as follows: In Section 2, the simulation tools are discussed:3D Ray Launching algorithm, as well as geometrical and dielectric model of tree. In Section 3, the simulation scenario is described and the obtained simulation results are shown. Finally, the comparison between these simulation results and the radio channel measurements shown in Reference [4] is made.

\section{Radio Channel Characterization of Vegetation Environments}

In this section, the used main elements required for performing the radio channel characterization are described: the simulation algorithm and the created geometrical and dielectric model of the trees.

\subsection{Simulation Software}

The most commonly radio propagation models used to characterize this type of complex environments are theoretical or empirical models. Theoretical methodologies offer rapid results but they lack precision. On the other hand, empirical techniques are more accurate but because of that, they require extensive measurement campaigns in the considered environment. Methods based on geometrical optics such as ray tracing or ray launching achieve a good trade-off between simulations accuracy and computational cost.

In this work, an in-house developed 3D Ray Launching (3D-RL) algorithm has been used to characterize inhomogeneous vegetation environments. The 3D-RL approach is divided in three steps:

1. The first step consists in the design and creation of a realistic scenario, considering all the obstacles and scatterers within it.

2. The second step is the simulation procedure, where a set of rays are launched from the transmitter with a specific angular and spatial resolution. All of the parameters of the paths that follow the rays are stored during simulation, until the rays achieve the maximum number of reflections or the maximum specified delay. Electromagnetic phenomena such as reflection, refraction and diffraction are considered.

3. Finally, the third step processes the data and provides accurate electromagnetic radio wave propagation results.

A detailed description of the algorithm can be found in Reference [5] and its validation for complex environments, in which the vegetation has a great impact, can be found in Reference [6]. It is worth noting that in addition to reflection and refraction electromagnetic phenomena, the 
presented 3D-RL algorithm has the possibility to include diffraction phenomenon in the simulations. For the presented simulations, diffraction has been activated.

\subsection{Model of Tree}

The Orgi Forest is a 77-hectares-forest which is included in the European Natura 2000 network. Natura 2000 is the largest network of protected areas in the world and it offers protection to most valuable and threatened species in Europe. In the case of Orgi, the oak (Quercus robur) is the main species protected. For the presented analysis, the radio propagation through both pines and oaks has been assessed. For that purpose, novel computational tree models have been developed for its use in the 3D-RL algorithm. The oak tree consisted of a solid trunk and a homogeneous mass of leaves. Meanwhile, the pine was modelled with homogeneous branches, with air in between. Both the widths and the heights of the trees are parameterized. Figure 1 shows the created tree models for oaks and trees and Table 1 shows the parameters of the materials included for the simulations.

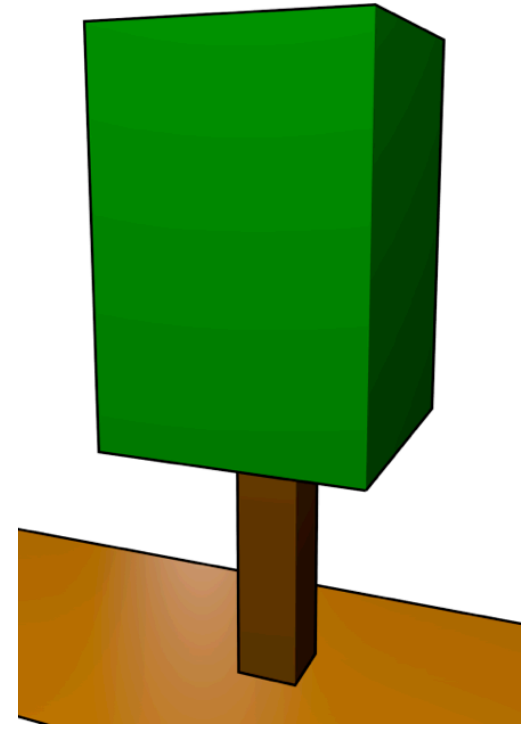

(a)

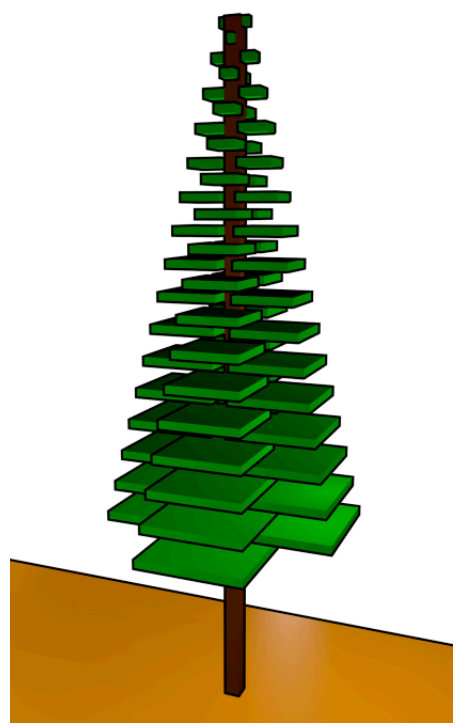

(b)

Figure 1. Created tree models for the 3D Ray Launching simulation tool. (a) Oak tree; (b) Pine tree.

Table 1. Material properties for Ray Launching simulations (at $2.4 \mathrm{GHz}$ ).

\begin{tabular}{ccc}
\hline Material & $\boldsymbol{\varepsilon}_{\mathbf{r}}$ & Conductivity $(\mathrm{S} / \mathbf{m})$ \\
\hline Foliage & 4.48 & 0.02 \\
Pine trunk & 1.97 & 0.052 \\
Oak trunk & 2.51 & 0.148 \\
Forest Ground & 4.8 & 0.98 \\
\hline
\end{tabular}

\section{Simulation Results}

The scenario simulates the transmissions between a static transmitter and a mobile terminal in the presence of a thick tree producing a signal blockage. This study considered two tree species: oak and pine. The scenario created for the simulations with the 3D-RL can be seen in Figure 2. The transmitting frequency used was $2.4 \mathrm{GHz}$. The transmitter, represented by a red circle (TX) has been placed at $2 \mathrm{~m}$ height. The entire bounding box (i.e., the walls of the scenario) has been defined as air with the aim of avoiding not desired multipath components, except the ground that has been defined with the material properties shown in Table 1. A summary of the simulation parameters is presented in Table 2. Although the 3D-RL tool provides results for the whole volume of the scenario, propagation losses corresponding to the yellow dashed line of Figure $2 b$ are analysed in this work. Specifically, linear paths at three heights have been considered: $1 \mathrm{~m}, 2 \mathrm{~m}$ and $3 \mathrm{~m}$. 


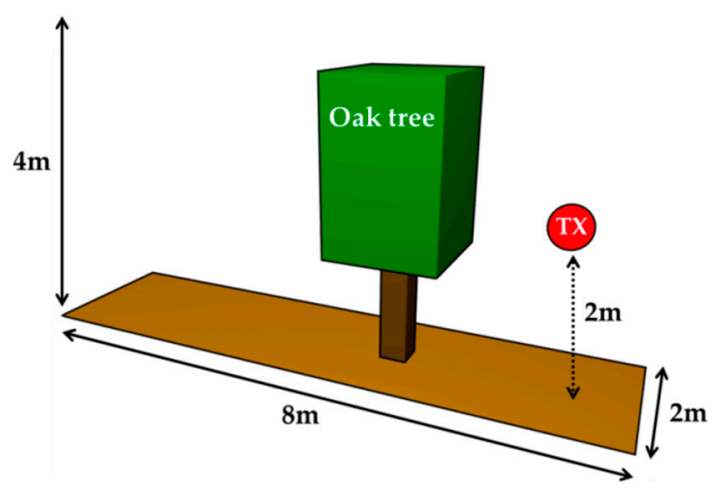

(a)

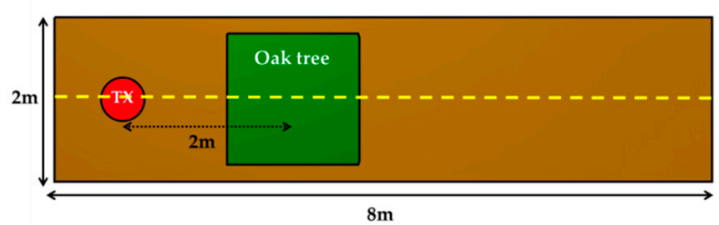

(b)

Figure 2. Created scenario for the 3D Ray Launching simulations. (a) General view; (b) Upper view.

Table 2. Ray Launching simulation parameters.

\begin{tabular}{cc}
\hline Parameters & Values \\
\hline Transmitted Power & $10 \mathrm{dBm}$ \\
Operation Frequency & $2.4 \mathrm{GHz}$ \\
Antenna Type/Gain & Monopole $/ 0 \mathrm{~dB}$ \\
Launched rays resolution & $1 \mathrm{degree}$ \\
Permitted maximum rebounds & 6 \\
Cuboids size (Mesh resolution) & $10 \mathrm{~cm} \times 10 \mathrm{~cm} \times 10 \mathrm{~cm}$ \\
\hline
\end{tabular}

\subsection{Path Loss}

The effect of the mobile receiver displacement on the path loss, moving from far to near the tree along the direct ray path direction, was analysed from the simulated data for both species of trees at 2.4 GHz. We concluded that the scattering produced by the tree can be divided into two zones: a diffraction dominant zone around in the vicinity of the tree followed by a free-space zone. The two different propagation situations can be identified in Figure 3. Far from the tree, the signal variation with distance fits the free-space power decay given that the line-of-sight (LOS) condition is dominant. However, as the receiver moves linearly toward the tree (actually the mass of leaves), the channel response varies from a response typical for a free-space scenario to a response typical for a multipath or scattering situation and the received signal starts a linear variation of opposite trend to the freespace. This would be the diffraction dominant zone. The diffraction produces that the large attenuation introduced by the tree blockage starts recovering and continue as a free-space component.

These results are similar to those found in Reference [4]. There it is described a scenario of a lowelevation, air-to-ground radio link blocked by an isolated tree with a transmitter placed over both the tree and a ground mobile receiver, for different species of trees at $\mathrm{X}$ band $(8-12 \mathrm{GHz})$ and $\mathrm{Ku}$ band (12-18 GHz) frequencies. However, apart from a free-space region, two different scattering zones were identified in Reference [4]: a diffuse scattering-dominant region close to the tree trunk, within which the signal level only admitted a model according to a statistical distribution function; and a colliding region wherein the diffraction on the tree crown prevails, modelled considering knife-edge diffraction loss with a correction of the tree height. Beyond this second scattering zone, the signal recovers the power decay corresponding to the free-space model.

In our model we have not clearly identified the zone corresponding to the diffuse scattering near the tree. This may be to the absence of air gaps in the model adopted for the mass of leaves which would turn the propagation media into a multi dispersive material. In Reference [4] the model was experimentally derived from measurements performed inside an anechoic chamber. For actual trees the mass of leaves is not homogeneous. The random fading effect of the leaves on the propagated signal contributes to produce the diffuse scattering zone. However, in Figure 3a, for oak, a noticeable effect is observed once the tree blockage ends: the signal attenuation decays sharply. This extreme 
attenuation may respond to the plausible existence of a diffuse scattering zone. Experimental measurements are needed to be carried in order to corroborate this fact. For the case of pine tree, the results are similar but less marked. It may be due to the fact that the pine tree model shows gaps of air that do not attenuate the signal as drastically as in the case of the homogeneous oak model.

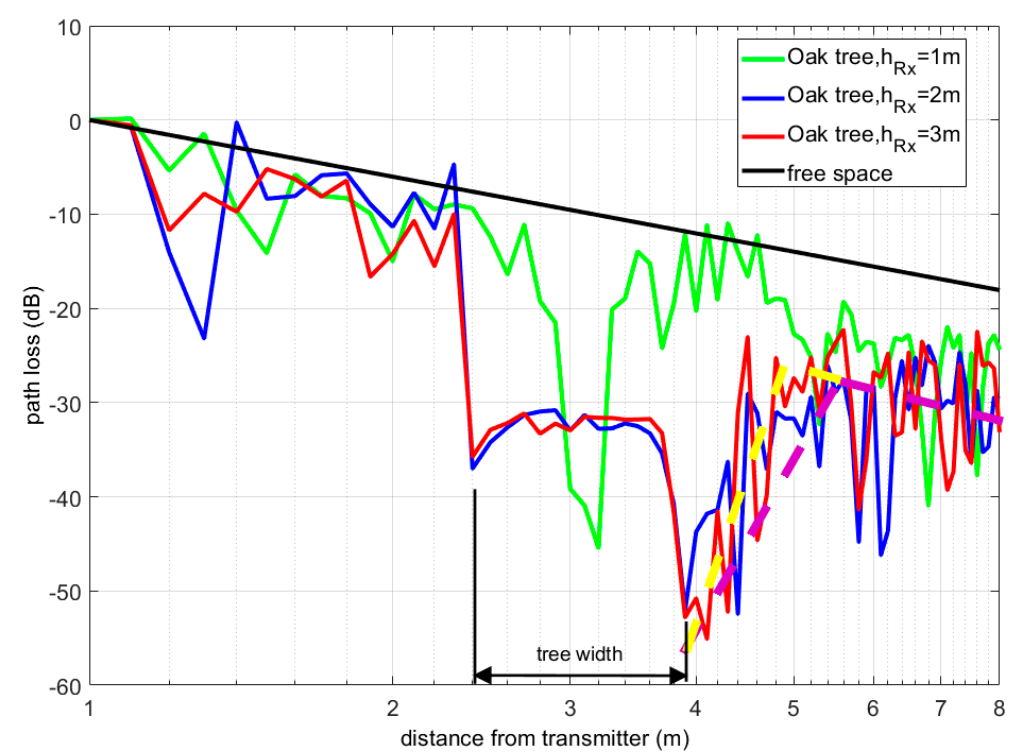

(a)

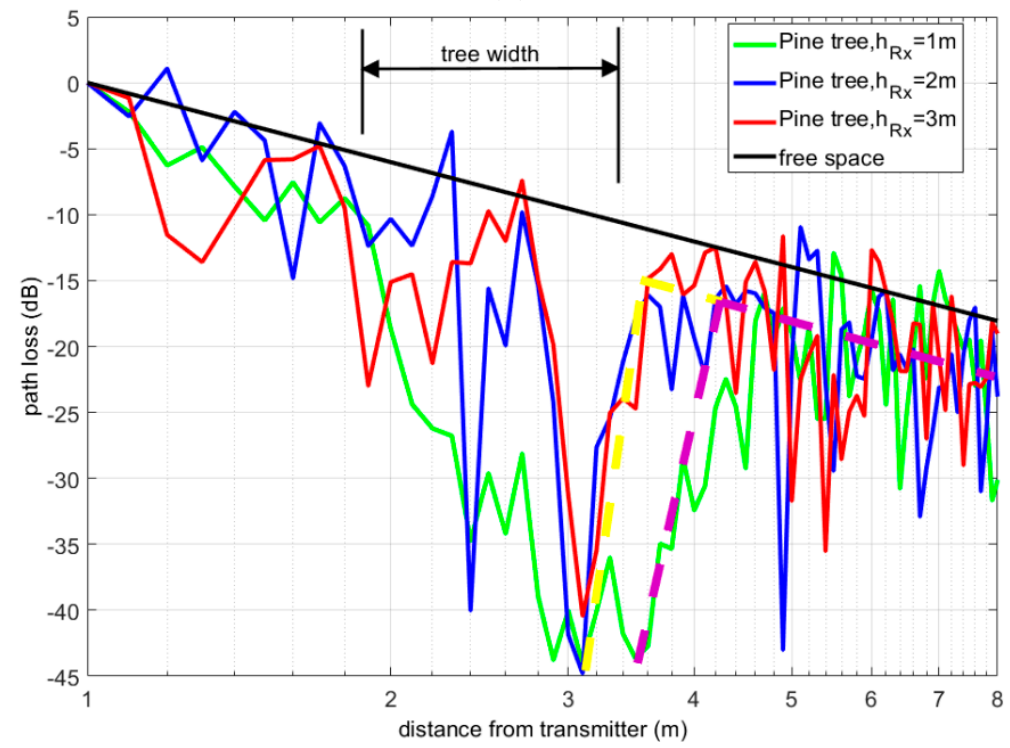

(b)

Figure 3. Simulation path loss for a single tree, (a) Oak tree; (b) Pine tree.

\subsection{Delay Spread}

As previously mentioned, models combining path loss and multipath propagation are rarely considered but the presented 3D Ray Launching algorithm can also provide multipath propagation information, as can be seen in Figure 4, where simulation results of the Delay Spread are shown for different heights of pine and oak tree models. As can be seen, at height $1 \mathrm{~m}$, the trunk is the influent part but for $3 \mathrm{~m}$ height the pine tree model presents too much air gaps (i.e., behaves like low density medium), while the oak tree model behaves like homogeneous medium. These facts will be studied in future works. Anyway, the obtained results are in accordance with $[7,8]$. 


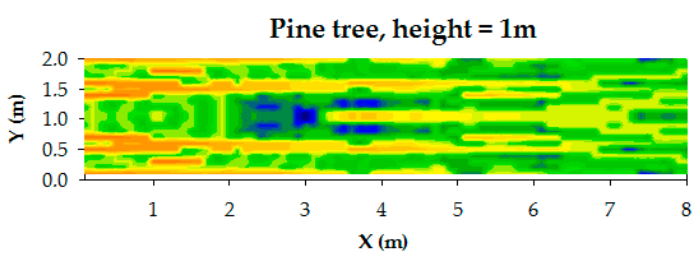

Pine tree, height $=3 \mathrm{~m}$

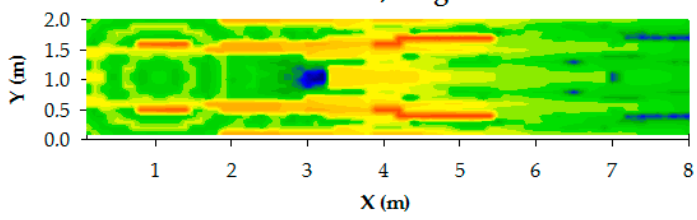

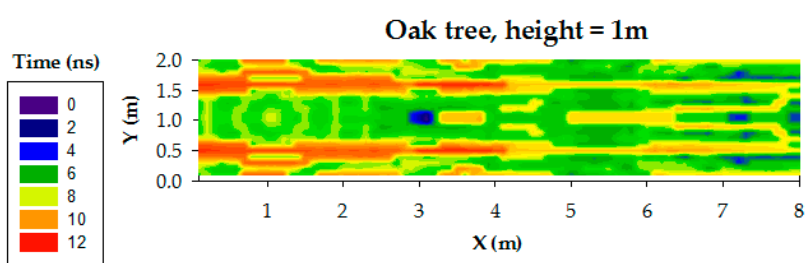

Oak tree, height $=3 \mathrm{~m}$

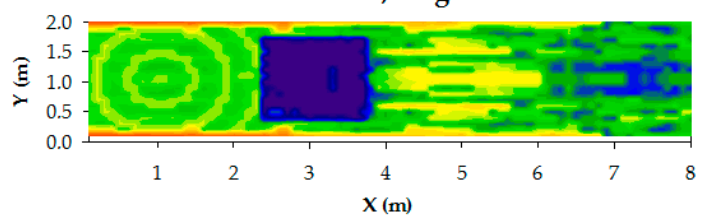

Figure 4. Simulation results of the Delay Spread, for both pine tree (left) and oak tree (right).

Author Contributions: Conceptualization, A.A.; Methodology, A.A., P.L.-I. and F.F.; Software and Simulations, P.L.-I., A.E., M.C.-E. and L.A.; writing-original draft preparation, A.A., L.A. and P.L.-I.; writing-review and editing, A.A., P.L.-I. and F.F.; Visualization, E.A., P.L.-I. and A.A.; Supervision, A.A.

Funding: This research was funded by Xunta de Galicia, grant GRC2015/19.

Conflicts of Interest: The authors declare no conflict of interest. The funders had no role in the design of the study; in the collection, analyses or interpretation of data; in the writing of the manuscript or in the decision to publish the results.

\section{References}

1. Klaina, H.; Vazquez Alejos, A.; Aghzout, O.; Falcone, F. Narrowband Characterization of Near-Ground Radio Channel for Wireless Sensors Networks at 5G-IoT Bands. Sensors 2018, 18, 2428.

2. The European Cooperation in Science and Technology, COST Action 235: Radiowave Propagation Effects on Next Generation Fixed Services Terrestrial Telecommunications Systems; Final Report; European Commission: Brussels, Belgium, 1996.

3. International Telecommunication Union. Recommendation ITU-R P.833-8: Attenuation in vegetation; United Nations: Geneva, Switzerland, 2013.

4. Cid, E.L.; Alejos, A.V.; Sanchez, M.G. Signaling Through Scattered Vegetation: Empirical Loss Modeling for Low Elevation Angle Satellite Paths Obstructed by Isolated Thin Trees. IEEE Veh. Technol. Mag. 2016, 11, 22-28.

5. Azpilicueta, L.; Rawat, M.; Rawat, K.; Ghannouchi, F.; Falcone, F. Convergence Analysis in Deterministic 3D Ray Launching Radio Channel Estimation in Complex Environments. ACES J. 2014, 29, 256-271.

6. Azpilicueta, L.; Lopez-Iturri, P.; Aguirre, E.; Mateo, I.; Astrain, J.J.; Villadangos, J.; Falcone, F. Analysis of Radio Wave Propagation for ISM 2.4 GHz Wireless Sensor Networks in Inhomogeneous Vegetation Environment. Sensors 2014, 14, 23650-23672.

7. Lang, R.H.; Schneider, A. Radiowave Propagation Within Trunk-dominated Forests: Coherence Bandwidth and Delay-spread. In Proceedings of the 12th Canadian Symposium on Remote Sensing Geoscience and Remote Sensing Symposium, Vancouver, BC, Canada, 10-14 July 1989; Volume 5, pp. 2847-2847.

8. Yang, F.-C. Forest Channel Characterization in the $5 \mathrm{GHz}$ Band. Master's Thesis, Russ College of Engineering and Technology of Ohio University, Athens, OH, USA, 2008. Available online: https://etd.ohiolink.edu/rws_etd/document/get/ohiou1226076756/inline (accessed on 15 October 2018). 